\title{
HIPERPARATIROIDISMO PRIMARIO Y EMBARAZO: REPORTE DE UN CASO
}

\author{
Ernesto Perucca P. 1, Eduardo Cuellar J. 1, Eliecer Pincheira U. ${ }^{4}$, Rocío Arenas P. 1 , \\ Eduardo Betancourt O. 4, Francisco Díaz M. 1, Giovanni Peña M. ${ }^{4}$, Eddy Delgado G. 4 , \\ Miguel González P. ${ }^{2}$, Fernando Uribe R. ${ }^{2}$, Jorge Castillo A. ${ }^{3}$, Moisés León V. ${ }^{a}$ \\ 1 Unidad de Medicina Materno Fetal, Servicio de Obstetricia y Ginecología; ${ }^{2}$ Servicio de Cirugía; ${ }^{3}$ Servicio de Anato- \\ mía Patológica, Hospital Barros Luco Trudeau. ${ }^{4}$ Programa de Postgrado en Obstetricia y Ginecología, Universidad de \\ Chile.
}

a Alumno, Escuela de Medicina, Universidad de Chile.

\section{RESUMEN}

El hiperparatiroidismo primario (HPTP) se caracteriza por una hipersecreción autónoma de paratohormona (PTH), lo que provoca hipercalcemia e hipercalciuria, con afectación ósea, renal y de otras partes del organismo, en grado variable. EI HPTP tiene una prevalencia de 1 en 1000 en la población general y se presenta con mayor frecuencia en mujeres que en hombres. Es infrecuente su presentación en el embarazo, y sus complicaciones derivadas de la hipercalcemia pueden afectar tanto a la madre como al feto. Presentamos el caso de una paciente de 37 años, cursando un embarazo de 10 semanas, que ingresó al servicio con los diagnósticos de nefrocalcinosis y pielonefritis aguda, a la que se diagnosticó un hiperparatiroidismo primario, cuya etiología fue un adenoma paratiroideo único.

PALABRAS CLAVE: Hiperparatiroidismo primario, embarazo

\section{SUMMARY}

Primary hyperparathyroidism (HPTP) is a disorder characterized by elevated levels of parathyroid hormone (PTH), which leads to hypercalcemia and hypercalciuria, with variable osseous, renal and other systems affectation. HPTP has a prevalence of 1:1000 in general population and is more frequent in women than men. It is very rare during pregnancy, and its complications are produced by hypercalcemia and they can affect the mother and the fetus. We present the case of a 37 year old woman, with a 10+2 weeks pregnancy that was admitted in our Hospital with nephrocalcinosis and urinary tract infection, whom was diagnosed primary hyperparathyroidism, whose etiology was a single parathyroid adenoma.

KEY WORDS: Primary hyperparathyroidism, pregnancy 


\section{INTRODUCCIÓN}

La homeostasis del calcio en el embarazo está adaptada a promover el aporte activo de 25 a 30 gramos de calcio al feto, conservando la estructura ósea materna $(1,2)$. Estos mecanismos de regulación son controlados principalmente por acción del calcio no-receptor específico y por varias hormonas, siendo la principal la paratohormona. $(1,2)$

El rol de la paratohormona (PTH) en el embarazo no esta bien definido, siendo sus acciones múltiples, como participar en la homeostasis del calcio, embriogénesis, la formación del esqueleto fetal y la regulación del calcio materno (1).

La hipercalcemia del embarazo puede tener varias etiologías, siendo la mas frecuente el hiperparatiroidismo primario (HPTP), definido como hipercalcemia e hipercalciuria persistente asociados a niveles de paratohormona elevados $(1,2,3)$.

El HPTP es una entidad muy poco frecuente $(1,3)$, se ha descrito una prevalencia de 1 en 1000 en la población general, afectando principalmente pacientes sobre los 45 años, siendo más frecuente en mujeres que en hombres $(2,4)$. Hunter \& Turnbull en 1931(5), reportaron el primer caso de HPTP durante el embarazo.

La mayoría de los casos de HPTP son diagnosticados como un hallazgo incidental durante un análisis de laboratorio rutinario, siendo asintomático en pacientes no embarazadas en el $50-80 \%(6,7)$. En embarazadas el tema es más controversial: Mestman (7), describe que durante el embarazo el $70 \%$ de las pacientes con HPTP son sintomáticas; contrariamente, existen publicaciones que afirman que la real incidencia del HPTP en embarazadas es incierta debido a que más del $80 \%$ de las gestantes con esta patología cursarían asintomáticas (8). Sin embargo, se ha visto que en las gestantes existe una amplia variedad de síntomas: gastrointestinales (anorexia, náuseas, vómitos), baja de peso, fatiga, dolor de cabeza e hipertensión. Pueden haber complicaciones, siendo las más frecuentes la nefrocalcinosis y nefrolitiasis, enfermedad ósea, pancreatitis, infección del tracto urinario a repetición, abortos espontáneos, hiperemesis gravídica, cambios mentales y crisis hipercalcémicas; aunque la mayoría de las veces se trata de sintomatología inespecífica y de intensidad moderada $(6,7,8)$. Las complicaciones fetales incluyen: RCIU, parto prematuro, óbito fetal, convulsiones e hipocalcemia neonatal $(8,9)$. Se ha descrito que la morbilidad materna y fetal es proporcional a la calcemia (10). En las gestantes, la causa más frecuente es el adenoma paratiroideo único, que causa el $80-85 \%$ de los casos $(2,8)$.

La cirugía constituye una alternativa eficaz y segura frente al tratamiento conservador (8). La primera paratiroidectomía fue realizada por Petit \& Clarck en 1947 (11).

Presentamos el caso de una embarazada que ingresa por pielonefritis aguda y antecedentes de nefrocalcinosis, en la cual se diagnostica un HPTP por adenoma único del paratiroides.

\section{Caso clínico}

Paciente de 37 años, multípara de 2 partos vaginales, el último en agosto del 2003, con antecedentes de nefrocalcinosis y nefromegalia diagnosticadas en 2004. Ingresa al Servicio de Obstetricia y Ginecología del Hospital Barros Luco-Trudeau el 15 de mayo de 2008, derivada desde el Hospital San Luis de Buin, cursando con un embarazo de 10 semanas por fecha de última menstruación acorde con ecografía precoz. Presenta compromiso del estado general de inicio reciente, nauseas, vómitos e intensa epigastralgia, diagnosticándose pielonefritis aguda. Se inicia tratamiento antibiótico con ceftriaxona y es trasladada a nuestro centro para completar estudio y manejo. Al ingreso destaca además, anemia ferropriva moderada e historia de larga evolución de baja de peso, astenia, adinamia y mialgias.

A su ingreso al servicio se continúa tratamiento antibiótico con cefalosporinas de primera generación (cefazolina) y evaluación por equipo de nefrología, quienes solicitan exámenes para estudio de su patología renal de base: PTH, calcemia, fosfemia, calciuria y función renal. Los resultados muestran presencia de hipercalcemia, hipercalciuria y aumento de la PTH, diagnosticándose HPTP. Se inicia hidratación parenteral abundante y se agrega furosemida, para evitar las complicaciones derivadas de la hipercalcemia.

Se solicita ecografía cervical que mostró nódulo hipoecogénico en la región posterior de la glándula tiroides, vascularizado al doppler, sin calcificaciones patológicas, de $1,3 \times 0,8 \mathrm{~cm}$, con tiroides de aspecto normal, sin evidencias de lesiones focales, ni adenopatías (Figura 1).

Se concluye adenoma paratiroideo izquierdo, siendo evaluada por los equipos de endocrinología y cirugía de cabeza y cuello, programándose su resolución quirúrgica y evaluación previa por anestesiología. La cirugía se realiza el 02 de junio de 2008, a las 13 semanas de embarazo, donde se logra extraer el adenoma paratiroideo y restos de timo branquiales cuya biopsia confirma el diagnóstico (Figuras 2 y 3), sin presentar incidentes durante la intervención. Posteriormente evolucionó en forma satisfactoria, con normalización de los niveles 
de calcio y PTH en sangre al segundo día postoperatorio (Tabla I). Es dada de alta 4 días después en buenas condiciones generales, con ecotomografía obstétrica que muestra feto vivo, de 13 semanas, con traslucencia nucal de $2,2 \mathrm{~mm}$ y hueso nasal presente.

Continuó en control en Policlínico de Alto Riesgo Obstétrico en buenas condiciones generales, con adecuado aumento de peso y niveles normales calcemia y PTH. Parto espontáneo el 19 de noviembre de 2008 a las 37 semanas de gestación, obteniéndose recién nacido vivo, sexo masculino, $2.974 \mathrm{~g}$, talla $48 \mathrm{~cm}$, Apgar 8-9.

\section{DISCUSIÓN}

La primera dificultad frente al HPTP está en establecer el diagnóstico, debido a que en la mayoría de los casos los síntomas son escasos y cuando están presentes son poco específicos. Si a esto se agrega la sintomatología propia del embarazo que puede enmascarar una hipercalcemia $(12,13)$,

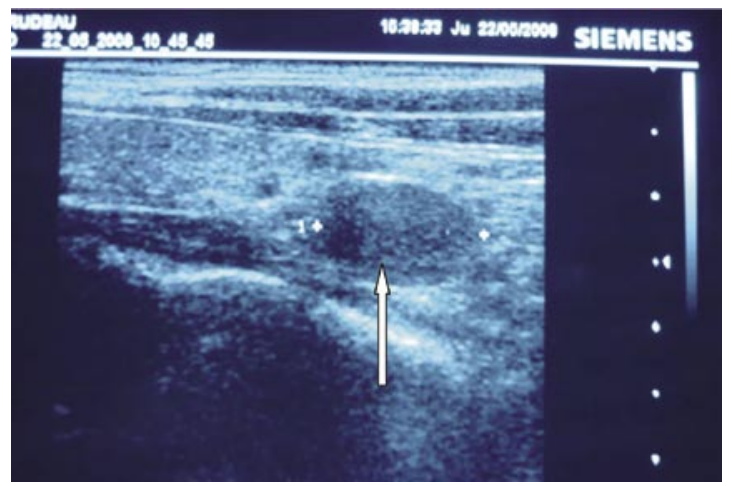

Figura 1. Ecotomografía de cuello. Se aprecia nódulo de paratiroides (flecha).

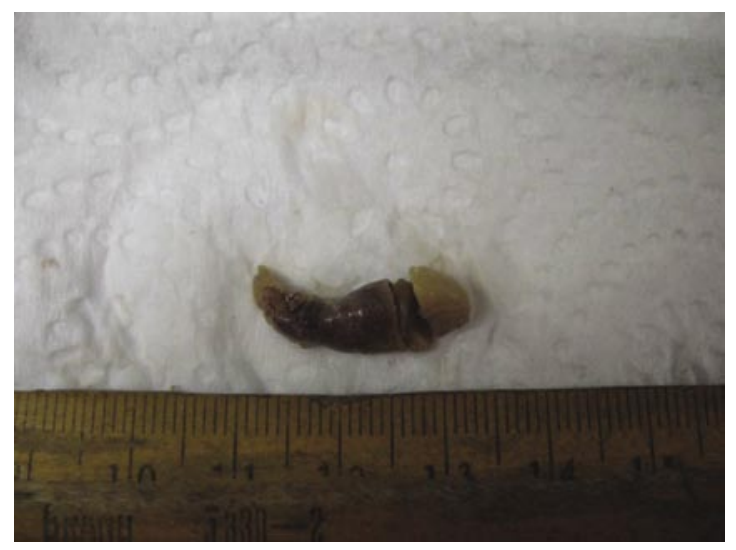

Figura 2. Pieza quirúrgica. se afronta un gran desafío en el proceso diagnóstico, por lo que el conocimiento de esta entidad por los profesionales de salud es clave a la hora de sospechar esta patología. En este caso la paciente presentaba sintomatología de larga evolución sugerente de hipercalcemia, que no llevaron al diagnóstico precoz, a pesar del antecedente de nefrocalcinosis.

Está descrito que el diagnóstico de HPTP se realiza frente a la presencia de hipercalcemia, niveles elevados de paratohormona e hipercalciuria mantenida (3), todos estos hallazgos encontrados en la paciente. Además, la etiología fue un adenoma paratiroideo único, concordando con lo descrito en la literatura $(2,8)$.

La ecografía cervical es la técnica de imagen más indicada, por contraindicación del uso de isótopos radiactivos en el embarazo $(8,14)$. En la actualidad, el tratamiento de elección es la remoción quirúrgica del adenoma paratiroideo realizado durante el segundo trimestre de embarazo (15).

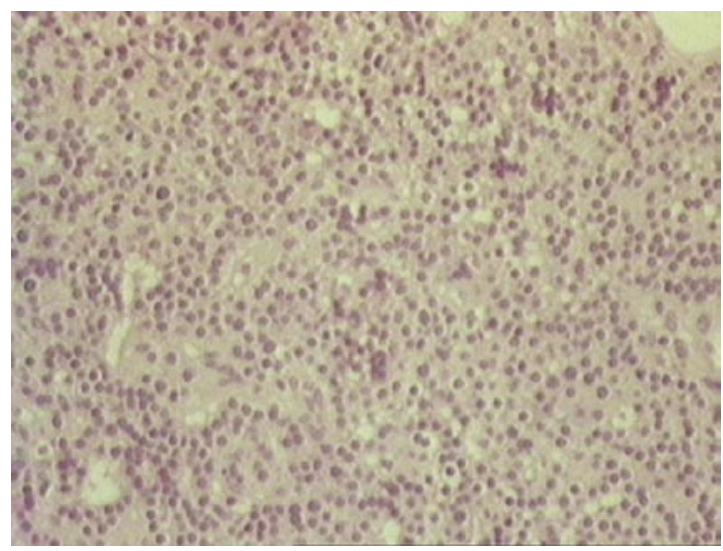

Figura 3. Histología de adenoma de paratiroides.

Tabla I

EVOLUCIÓN DE LA CONCENTRACIÓN DEL CALCIO, FÓSFORO Y PTH EN PLASMA Y VALORES DE REFERENCIA

\begin{tabular}{llll}
\hline Parámetro & $\begin{array}{l}\text { Ingreso } \\
(16 / 05 / 08)\end{array}$ & $\begin{array}{l}\text { Alta } \\
(04 / 06 / 08)\end{array}$ & $\begin{array}{l}\text { Valor de } \\
\text { referencia }\end{array}$ \\
\hline Calcio $(\mathrm{mg} / \mathrm{dL})$ & 14,3 & 11,9 & $8,5-10,2$ \\
Fósforo $(\mathrm{mg} / \mathrm{dL})$ & 1,0 & 2,4 & $2,7-4,5$ \\
PTH $(\mathrm{pg} / \mathrm{dL})$ & 262 & 6,6 & $15-65$ \\
\hline
\end{tabular}


El manejo conservador con hidratación abundante, dieta normocalcémica y furosemida esta indicado solamente mientras se programa el tratamiento quirúrgico definitivo (14). Otros fármacos utilizados para el manejo de la hipercalcemia como calcitonina o bifosfonatos no están recomendados durante el embarazo (10).

Destacamos la revisión de Nelly (16), en su estudio se concluye que las pacientes que presentaban HPTP y que recibían tratamiento conservador tenían una mayor tasa de complicaciones neonatales, que las que habían recibido tratamiento quirúrgico. Además, estos autores recomendaban la realización de paratiroidectomía en el segundo trimestre del embarazo, tal como se realizó en nuestra paciente. El tratamiento quirúrgico en el primer trimestre de la gestación aumenta el riesgo de daño teratogénico fetal derivado de la anestesia, mientras que, si se efectúa en el tercer trimestre, se incrementa el riesgo de parto de pretérmino (16). Schnatz y cols (8), establecen una serie de indicaciones quirúrgicas en las gestantes que se presentan en la Tabla II.

\section{CONCLUSIÓN}

El HPTP es una entidad poco frecuente que

\section{Tabla II \\ INDICACIONES QUIRÚRGICAS EN PACIENTES EMBARAZADAS (8)}

1. Síntomas de hiperparatiroidismo primario

2. Elevación del calcio sérico $>12 \mathrm{mg} / \mathrm{dl}$

3. Antecedente de crisis hipercalcémica con riesgo vital

4. Reducción del clearance de creatinina $>30 \%$, de acuerdo a edad y embarazo, sin otra causa que lo explique

5. Presencia de nefrolitiasis o nefrocalcinosis

6. Aumento en la excreción urinaria de calcio: $>400 \mathrm{mg}$ de calcio en orina de 24 horas

7. Evidencia de compromiso óseo

8. Sospecha de incumplimiento o inconsistencia en el seguimiento

9. Coexistencia de enfermedades que pueden complicar la evolución de la enfermedad o el manejo posterior

10. Paciente que solicita cirugía puede causar morbimortalidad importante tanto a la gestante como al feto. Es de difícil diagnóstico, por lo que requiere que sea una patología conocida por especialistas y médicos en general, para así poder realizar una intervención en el lugar y tiempo adecuado.

\section{BIBLIOGRAFÍA}

1. Kohlmeier L, Marcus R. Calcium Disorders of Pregnancy. Endocrinol Metab Clin North Am 1995;24(1):1539.

2. Eigelberger MS, Clark OH. Surgical approaches to primary hyperparathyroidism. Endocrinol Metab Clin North Am 2000;29(3):479-502.

3. Carella MJ, Gossain VV. Hyperparathyroidism and pregnancy. Case report and review. J Gen Intern Med 1992;7(4):448-53.

4. Schneider B, Peschgens T, Hörnchen H, Schild R, Kutta T. Primary hyperparathyroidism in the $3 \mathrm{~d}$ pregnancy trimester. Dtsch Med Wochenschr 1995;120(33):11236.

5. Hunter D, Turnbull H. Hyperparathyroidism: generalised osteitis fibrosa with observation upon bones, parathyroid tumor and the normal parathyroid gland. $\mathrm{Br} \mathrm{J}$ Surg 1931;19:203-6.

6. Ficinski ML, Mestman JH. Primary hyperparathyroidism during pregnancy. Endocr Pract 1996;2(5):3627.

7. Mestman JH. Parathyroid disorders of pregnancy. Semin Perinatol 1998; 22(6):485-96.

8. Schnatz PF, Curry SL. Primary hyperparathyroidism in pregnancy: Evicence - Based management. Obstet Gynecol Surv 2002; 57(6):365-76.

9. Graham EM, Freedman LJ, Forouzan I. Intrauterine growth retardation in a woman with primary hyperparathyroidism: a case report. J Reprod Med 1998;43(5):451-4.

10. Shangold MM, Dor N, Welt SI, Fleischman AR, Crenshaw MC Jr. Hyperparathyroidism and pregnancy. a review. Obstet Gynecol Surv 1982;37(4):217-28.

11. Petit DW, Clark RL. Hyperparathyroidism and pregnancy. Am J Surg 1947;74:860.

12. Torres I, Carral F, García A, Aguilar M. Primary hiperparathyroidism and pregnancy. Endocrinología 2003;50:175-7.

13. Molitch ME. Endocrine emergencies in pregnancy. Baillieres Clin Endocrinol Metab 1992;6(1):167-91.

14. National Institutes of Health. Consensus development conference statement on primary hyperparathyroidism. J Bone Miner Res 1991; 6:S9-13.

15. Rooney DP, Traub AI, Russell CF, Hadden DR. Cure of hyperparathyroidism in pregnancy by sternotomy and removal of a mediastinal parathyroid adenoma. Postgrad Med J 1998;74(870):233-4.

16. Kelly TR. Primary hyperparathyroidism during pregnancy. Surgery 1991; 110(6):1028-34. 